Bull. Austral. Math. Soc.

Vol. 63 (2001) [133-140]

\title{
RELATIONSHIPS BETWEEN WIDTHS OF A CONVEX BODY AND OF AN INSCRIBED PARALLELOTOPE
}

MAREK LASSAK

\begin{abstract}
Assume that a parallelotope $P$ is inscribed in a three-dimensional convex body $C$. A conjecture says that $w_{1}^{-1}+w_{2}^{-1}+w_{3}^{-1} \geqslant 1$, where $w_{i}$ is the ratio of the width of $C$ to the width of $P$ for the direction perpendicular to the $i$-th pair of parallel facets of $P$. We prove three weaker inequalities. One of them is $w_{1}^{-1}+w_{2}^{-1}+a_{3}^{-1} \geqslant 1$, where $a_{3}$ denotes the related axial diameter of $C$.
\end{abstract}

Let $C$ be a convex body in Euclidean $n$-space $E^{n}$ and let $P$ be a parallelotope inscribed in $C$. Denote by $w_{i}$ the ratio of the width of $C$ to the width of $P$ for the direction perpendicular to the $i$-th pair of parallel facets of $P$, for $i=1, \ldots, n$. We recall a conjecture from [2].

CONJECTURE 1. For every convex body $C \subset E^{n}$ and every parallelotope $P$ inscribed in $C$ we have

$$
\sum_{i=1}^{n} w_{i}^{-1} \geqslant 1 .
$$

For $n=2$ Conjecture 1 holds true as shown in [2]. The note [1] announces without a proof that Conjecture 1 is true for $n=3$ under some very specific assumptions.

We consider the three-dimensional case. We have $P=E_{1}+E_{2}+E_{3}$, where $E_{1}$, $E_{2}, E_{3}$ are three independent edges of $P$. Of course, $F_{1}=E_{2}+E_{3}, F_{2}=E_{1}+E_{3}$ and $F_{3}=E_{1}+E_{2}$ are three non-parallel facets of $P$.

We define the $i$-th axial diameter of $C$ as the ratio $a_{i}$ of the length of the longest segment in $C$ which is parallel to $E_{i}$ to the length of $E_{i}$, where $i=1,2,3$. In other words, $a_{i}$ is the length of the longest segment in $C$ parallel to the $i$-th axis of a coordinate system whose unit vectors determine the edges $E_{1}, E_{2}$ and $E_{3}$. The term axial diameter was introduced by $\operatorname{Scott}[3,4]$. If $P$ is the unit cube then $a_{i}$ and $w_{i}$ are just the inner and outer 1-quermasses of $C$.

Received 2nd May, 2000

Research supported by Deutsche Forschungsgemainschaft and provided at Freie Universität Berlin.

Copyright Clearance Centre, Inc. Serial-fee code: 0004-9727/01 \$A2.00+0.00. 
Since $a_{i} \leqslant w_{i}$ for $i=1,2,3$, the inequality $w_{1}^{-1}+w_{2}^{-1}+w_{3}^{-1} \geqslant 1$ is stronger than $a_{1}^{-1}+a_{2}^{-1}+a_{3}^{-1} \geqslant 1$. The last inequality has been proved by Scott [3]. It has been improved up to $w_{1}^{-1}+a_{2}^{-1}+a_{3}^{-1} \geqslant 1$ by Wills [5]. The results of Scott and Wills hold true for the more general situation of a convex body without interior lattice points.

Our aim is to prove a few inequalities which are better than the inequality of Scott but still weaker than the conjectured inequality (1).

Theorem 1. Assume that a parallelotope $P$ is inscribed in a three-dimensional convex body $C$. Then

$$
w_{1}^{-1}+w_{2}^{-1}+a_{3}^{-1} \geqslant 1
$$

ProOF: We do not make our considerations narrower assuming that $P$ is a cube with vertices $\left(e_{1}, e_{2}, e_{3}\right)$, where $e_{i} \in\{-1,1\}$ for $i=1,2,3$, in a rectangular coordinate system. We can always obtain this case by an affine transformation.

We apply Steiner symmetrisation with respect to the plane $x_{3}=0$. Denote by $D$ the image of our body $C$. Of course, $w_{1}$ and $w_{2}$ remain unchanged. Let $H_{i}, H_{i}^{\prime}$ be the supporting planes of $D$ perpendicular to $E_{i}$, where $i=1,2$. Denote be $L_{i}$ and $L_{i}^{\prime}$ the intersection of the plane $x_{3}=0$ with $H_{i}$ and $H_{i}^{\prime}$, respectively, where $i=1,2$. Observe that $H_{i}$ and $H_{i}^{\prime}$ touch $D$ at some points which are in $L_{i}$ and $L_{i}^{\prime}$, respectively, where $i=1,2$. Of course, the width of $D$ in the direction perpendicular to the plane $x_{3}=0$ is $a_{3}$. Let $g$ be the point of support of $D$ by the supporting plane $x_{3}=a_{3} / 2$.

Consider the homothety $\gamma$ with centre $g$ and ratio $1-a_{3}^{-1}$. The image of the plane $x_{3}=0$ is the plane $x_{3}=1$. The common part $C_{0}$ of $C$ and of the plane $x_{3}=1$ is a two-dimensional convex body in the plane $x_{3}=1$. Since the cube $P$ is inscribed in $C$, the square face $P_{0}$ of $P$ which lies in the plane $x_{3}=1$ is inscribed in $C_{0}$. Denote by $v_{1}$ and $v_{2}$ the widths of $C_{0}$ in the directions perpendicular to the sides of $P_{0}$; the directions are perpendicular to $H_{1}$ and $H_{2}$, respectively. Since the two-dimensional case of Conjecture 1 holds true (see [2, Lemma]), we have $v_{1}^{-1}+v_{2}^{-1} \geqslant 1$. On the other hand, the width of the strip between the pair of parallel straight lines $\gamma\left(L_{i}\right), \gamma\left(L_{i}^{\prime}\right)$ is $w_{i}\left(1-a_{3}^{-1}\right)$, where $i=1,2$. Since the straight lines $L_{1}, L_{1}^{\prime}, L_{2}, L_{2}^{\prime}$ have nonempty intersections with $C$, from the convexity of $C$ it follows that the straight lines $\gamma\left(L_{1}\right), \gamma\left(L_{1}^{\prime}\right), \gamma\left(L_{2}\right), \gamma\left(L_{2}^{\prime}\right)$ have non-empty intersections with $C_{0}$. Consequently, $w_{1}\left(1-a_{3}^{-1}\right) \leqslant v_{1}$ and $w_{2}\left(1-a_{3}^{-1}\right) \leqslant v_{2}$. So we have $\left(w_{1}\left(1-a_{3}^{-1}\right)\right)^{-1}+\left(w_{2}(1-\right.$ $\left.\left.a_{3}^{-1}\right)\right)^{-1} \geqslant 1$. Thus $w_{1}^{-1}+w_{2}^{-1} \geqslant 1-a_{3}^{-1}$. Hence $w_{1}^{-1}+w_{2}^{-1}+a_{3}^{-1} \geqslant 1$.

Wills [5] presents an example that (2) does not hold true in the more general situation when $C$ is a convex body without points of an integer lattice in the interior.

When we change the roles of the axes in Theorem 2 , we get $w_{1}{ }^{-1}+a_{2}{ }^{-1}+w_{3}{ }^{-1} \geqslant 1$ and $a_{1}{ }^{-1}+w_{2}{ }^{-1}+w_{3}^{-1} \geqslant 1$. Adding those three inequalities and dividing by 3 
we obtain the following corollary which, in a sense, moves us two thirds of the way from the Scott's inequality $a_{1}{ }^{-1}+a_{2}{ }^{-1}+a_{3}{ }^{-1} \geqslant 1$ to the conjectured inequality $w_{1}{ }^{-1}+w_{2}^{-1}+w_{3}^{-1} \geqslant 1$.

CoRollary 1. Assume that a parallelotope $P$ is inscribed in a three-dimensional convex body $C$. Then

$$
\frac{2}{3}\left(w_{1}^{-1}+w_{2}^{-1}+w_{3}^{-1}\right)+\frac{1}{3}\left(a_{1}^{-1}+a_{2}^{-1}+a_{3}^{-1}\right) \geqslant 1 .
$$

We note that the approach of the proof of Theorem 2 and induction arguments lead to the analogous inequality $w_{1}{ }^{-1}+w_{2}{ }^{-1}+a_{3}{ }^{-1}+\cdots+a_{n}{ }^{-1} \geqslant 1$ for an $n$-dimensional convex body with an inscribed parallelotope. In analogy to Corollary 1 , we also obtain $(2 / n) \sum_{i=1}^{n} w_{i}^{-1}+((n-2) / n) \sum_{i=1}^{n} a_{i}^{-1} \geqslant 1$.

By the $i$-th column $K_{i}$ generated by $P$, where $i \in\{1,2,3\}$, we understand the union of all straight lines parallel to $E_{i}$ which have non-empty intersection with $P$. Of course, $P=K_{1} \cap K_{2} \cap K_{3}$. Consider the ratio $k_{i}$ of the width of $C \cap K_{i}$ to the width of $P$ in the direction perpendicular to $F_{i}$, where $i=1,2,3$. Of course, $k_{i}$ is the outer 1 -quermass of $K_{i} \cap C$ in the direction of $E_{i}$.

Observe that if $C$ is centrally symmetric, then there is no longer segment in $C$ in the direction of $E_{i}$ than the segment being the intersection of $C$ with the straight line passing through the centres of $P$ and of $F_{i}$. Thus if $C$ is centrally symmetric, we have $a_{i} \leqslant k_{i} \leqslant w_{i}$ for $i=1,2,3$. Consequently, the inequality $k_{1}^{-1}+k_{2}^{-1}+k_{3}^{-1} \geqslant 1$ is stronger than $a_{1}^{-1}+a_{2}^{-1}+a_{3}^{-1} \geqslant 1$ but weaker than $w_{1}^{-1}+w_{2}^{-1}+w_{3}^{-1} \geqslant 1$.

Theorem 2. Assume that a parallelotope $P$ is inscribed in a three-dimensional centrally-symmetric convex body $C$. Then

$$
k_{1}^{-1}+k_{2}^{-1}+k_{3}^{-1} \geqslant 1 \text {. }
$$

Proof: As in the proof of Theorem 1, assume that $P$ is a cube with vertices $\left(e_{1}, e_{2}, e_{3}\right)$.

Of course, if at least one of the numbers $k_{1}, k_{2}, k_{3}$ is equal to 1 , then (3) holds true. Let

$$
k_{1}>1, k_{2}>1, k_{3}>1 \text {. }
$$

The assumption of our Theorem implies that the boundary of $C$ contains points $x=\left(x_{1}, x_{2}, k_{3}\right), y=\left(y_{1}, k_{2}, y_{3}\right), z=\left(k_{1}, z_{2}, z_{3}\right)$ such that all the coordinates $x_{1}, x_{2}, y_{1}, y_{3}, z_{2}, z_{3}$ are between -1 and 1 . What is more, we can assume that $x_{1}$ and $x_{2}$ are non-negative. (We can choose the order and the orientation of the axes of 
our coordinate system which ensures that.) From the central symmetry we see that the boundary of $C$ contains also points $-x,-y,-z$ symmetric with respect to the origin to $x, y, z$. There are eight triples of points from amongst $x, y, z,-x,-y,-z$ such that exactly one point in each triple is selected from each of the sets $\{x,-x\},\{y,-y\}$, $\{z,-z\}$. From (4) we see that our six points $x,-x, y,-y, z,-z$ are pairwise different. Thus each triple $\left\{e_{1} x, e_{2} y, e_{3} z\right\}$ of points, where $e_{1}, e_{2}, e_{3} \in\{1,2\}$, determines exactly one plane $\Pi\left\{e_{1} x, e_{2} y, e_{3} z\right\}$ passing through them. We obtain eight planes; four pairs of symmetric planes.

We shall prove our Theorem by contradition. In other words, we assume now that

$$
k_{1}^{-1}+k_{2}^{-1}+k_{3}^{-1}<1
$$

and our aim is to show that $P$ is not inscribed in $C$.

In order to prove that $P$ is not inscribed in $C$ it is sufficient to show that at least one of the eight vertices $\left(e_{1}, e_{2}, e_{3}\right)$ of $P$ is not in the boundary of $C$. We shall prove this by showing that at least one of the eight planes $\Pi\left\{e_{1} x, e_{2} y, e_{3} z\right\}$ has empty intersection with $P$. Taking into account the central symmetry of $C$ and $P$, it is sufficient to show that at least one of the following four determinants is positive:

$$
\begin{array}{ll}
d_{1}=\operatorname{det}\left[\begin{array}{lll}
1-x_{1} & 1-x_{2} & 1-k_{3} \\
1-y_{1} & 1-k_{2} & 1-y_{3} \\
1-k_{1} & 1-z_{2} & 1-z_{3}
\end{array}\right], & d_{2}=\operatorname{det}\left[\begin{array}{lll}
1+x_{1} & 1+x_{2} & 1-k_{3} \\
1-y_{1} & 1-k_{2} & 1+y_{3} \\
1-k_{1} & 1-z_{2} & 1+z_{3}
\end{array}\right], \\
d_{3}=\operatorname{det}\left[\begin{array}{lll}
1-x_{1} & 1+x_{2} & 1-k_{3} \\
1+y_{1} & 1-k_{2} & 1+y_{3} \\
1-k_{1} & 1+z_{2} & 1-z_{3}
\end{array}\right], & d_{4}=\operatorname{det}\left[\begin{array}{lll}
1+x_{1} & 1-x_{2} & 1-k_{3} \\
1+y_{1} & 1-k_{2} & 1-y_{3} \\
1-k_{1} & 1+z_{2} & 1+z_{3}
\end{array}\right] .
\end{array}
$$

Let

$$
\begin{aligned}
p & =k_{3}\left(y_{1}+z_{2}\right)-x_{1} y_{3}-x_{2} z_{3}, \\
q & =k_{2}\left(x_{1}+z_{3}\right)-x_{2} y_{1}-y_{3} z_{2}, \\
r & =k_{1}\left(x_{2}+y_{3}\right)-x_{1} z_{2}-y_{1} z_{3} \\
k & =k_{1} k_{2} k_{3}-k_{1} k_{2}-k_{1} k_{3}-k_{2} k_{3} \\
t & =-\left(k_{1}-1\right) x_{2} y_{3}-\left(k_{2}-1\right) x_{1} z_{3}-\left(k_{3}-1\right) y_{1} z_{2}+x_{1} y_{3} z_{2}+x_{2} y_{1} z_{3} .
\end{aligned}
$$

Since $k=k_{1} k_{2} k_{3}\left(1-k_{1}^{-1}-k_{2}^{-1}-k_{3}^{-1}\right)$, from (5) we see that $k>0$. A simple calculation shows that

$$
\begin{aligned}
& d_{1}=p+q+r+k+t \\
& d_{2}=p-q-r+k+t \\
& d_{3}=-p+q-r+k+t \\
& d_{4}=-p-q+r+k+t .
\end{aligned}
$$


Remember that $x_{1} \geqslant 0$ and $x_{2} \geqslant 0$. Each of the numbers $y_{1}, y_{3}, z_{2}, z_{3}$ can be nonnegative (which we mark by + ) or negative (marked by - ). We have $2^{4}=16$ cases in which each of the numbers $y_{1}, y_{3}, z_{2}, z_{3}$ is of a fixed sign. Some of those cases are equivalent in the following sense. We can change by rotation the coordinate system such that the oriented axes exchange their positions. But we allow only such an exchange after which the new $x_{1}$ and $x_{2}$ are again non-negative. It is easy to see that we have exactly 6 non-equivalent cases. They are considered in Cases 1-6 below. Successive signs + or - mean that successive numbers $x_{1}, x_{2}, y_{1}, y_{3}, z_{2}, z_{3}$ are non-negative or negative, respectively.

In every case, we show that at least one of the determinants $d_{1}, d_{2}, d_{3}, d_{4}$ is positive. In order to conclude this, we show that a positive linear combination of those four determinants is positive (sometimes the combination is reduced to a single determinant). In every case (besides Case 1), and subcase we present our linear combination as a sum of non-negative components, from which at least one is positive. This positive component is always equal or greater than $\delta k$, where $\delta>0$. We remember that $k>0$. The positiveness of $\delta k$ and the non-negativeness of the remaining components follows always from the assumptions of the considered case (or subcase).

CASE 1. ++++++ . Of course, if $x_{1}=x_{2}=y_{1}=y_{3}=z_{2}=z_{3}=0$, then $d_{1}=k>0$. Moreover, the first derivatives of $d_{1}$ with respect to each of the six variables are non-negative when the variables are at most 1 (for example, the derivative with respect to $x_{1}$ is $\left.\left(1-y_{3}\right)\left(1-z_{2}\right)-\left(1-k_{2}\right)\left(1-z_{3}\right) \geqslant 0\right)$. Thus $d_{1}>0$ in this case.

CASE 2. ++++-+ . We have $2 d_{1}+d_{3}+d_{4}=2 q+2 r+4 k+4 t=2\left(k_{1}-1\right)$ $\left(x_{2}+y_{3}-2 x_{2} y_{3}\right)+2 x_{2}\left(1-y_{1}\right)+2 y_{3}-2 x_{1} z_{2}\left(1-y_{3}\right)-2 y_{3} z_{2}\left(1-x_{1}\right)+2 k_{2} x_{1}\left(1-z_{3}\right)+$ $2\left(k_{2}-1\right) z_{3}\left(1-x_{1}\right)+2 z_{3}\left(1-y_{1}\right)+2 x_{1} z_{3}-4\left(k_{3}-1\right) y_{1} z_{2}+4 x_{2} y_{1} z_{3}+4 k>0$.

CASE 3. ++++-- . Of course, $d_{1}+d_{4}=2 r+2 k+2 t=2 k_{1}\left(x_{2}+y_{3}-x_{2} y_{3}\right)+$ $2 x_{2} y_{3}-2\left(k_{2}-1\right) x_{1} z_{3}-2\left(k_{3}-1\right) y_{1} z_{2}+2 x_{1} z_{2}\left(y_{3}-1\right)+2 y_{1} z_{3}\left(x_{2}-1\right)+2 k>0$.

CASE 4. ++-+-+ . In view of (5) we have $1-k_{1}^{-1}-k_{2}^{-1}-k_{3}^{-1}>0$ and also we have $\left(3-k_{1}^{-1}-k_{2}^{-1}-k_{3}^{-1}\right) k_{1} k_{2} k_{3}-\left(k_{2}-1\right) x_{1} z_{3}-\left(k_{1}-1\right) x_{2} y_{3}-\left(k_{3}-1\right) y_{1} z_{2} \geqslant 2 k_{1} k_{2} k_{3}-$ $\left(k_{2}-1\right)-\left(k_{1}-1\right)-\left(k_{3}-1\right) \geqslant 2 k_{1} k_{2} k_{3}-k_{1} k_{2}-k_{2} k_{3}-k_{2} k_{3}+3=k+k_{1} k_{2} k_{3}+3>0$. Those two inequalities are used below, where we show that a positive linear combination of $d_{1}, d_{2}, d_{3}$ is positive: $\left(1-\left(k_{1}^{-1} / 2\right)-\left(k_{2}^{-1} / 2\right)\right) d_{1}+\left(1-\left(k_{2}^{-1} / 2\right)-\left(k_{3}^{-1} / 2\right)\right) d_{3}+$ $\left(1-\left(k_{1}^{-1} / 2\right)-\left(k_{3}^{-1} / 2\right)\right) d_{4}=\left(k_{2}-1\right)\left(x_{1}+z_{3}-2 x_{1} z_{3}\right)+\left(k_{1}-1\right)\left(x_{2}+y_{3}-2 x_{2} y_{3}\right)+$ $\left(k_{3}-1\right)\left(-y_{1}-z_{2}-2 y_{1} z_{2}\right)+\left(1-k_{1}^{-1}-k_{2}^{-1}-k_{3}^{-1}\right)\left[\left(3-k_{1}^{-1}-k_{2}^{-1}-k_{3}^{-1}\right) k_{1} k_{2} k_{3}-\right.$ $\left.\left(k_{2}-1\right) x_{1} z_{3}-\left(k_{1}-1\right) x_{2} y_{3}-\left(k_{3}-1\right) y_{1} z_{2}\right]+\left(1-k_{1}^{-1}\right)\left[x_{1} z_{2}\left(y_{3}-1\right)+y_{1} z_{3}\left(x_{2}-1\right)\right]+$ $\left(1-k_{2}^{-1}\right)\left[x_{2} y_{1}\left(z_{3}-1\right)+y_{3} z_{2}\left(x_{1}-1\right)\right]+\left(1-k_{3}^{-1}\right)\left[x_{1} y_{3}\left(z_{2}+1\right)+x_{2} z_{3}\left(y_{1}+1\right)\right]>0$.

CASE 5. ++-+- . We have $w=-\left(k_{1}-1\right) x_{2} y_{3}-\left(k_{2}-1\right) x_{1} z_{3}-\left(k_{3}-1\right) y_{1} z_{2} \geqslant 0$. 
SUBCASE 5.1. When $y_{1}+z_{2} \geqslant 0$ or when $x_{1}+z_{3} \geqslant 0$. The first inequality implies $d_{1}+d_{2}=2 p+2 k+2 t=2 w+2 x_{1} y_{3}\left(z_{2}-1\right)+2 x_{2} z_{3}\left(y_{1}-1\right)+2 k_{3}\left(y_{1}+z_{2}\right)+2 k>0$. Similarly, in the second possibility we obtain that $d_{1}+d_{3}>0$.

SUBCASE 5.2. When $x_{2}+y_{3} \leqslant 0$. We obtain that $d_{2}+d_{3}=-2 r+2 k+2 t=$ $2 w+2 x_{1} z_{2}\left(y_{3}+1\right)+2 y_{1} z_{3}\left(x_{2}+1\right)-2 k_{1}\left(x_{2}+y_{3}\right)+2 k>0$.

SUBCASE 5.3. When $y_{1}+z_{2}<0, x_{1}+z_{3}<0$ and $x_{2}+y_{3}>0$. The assumptions of this subcase mean that $z_{2}<-y_{1}, x_{1}<-z_{3}$ and $-y_{3}<x_{2}$. Since all the six numbers are positive, we obtain $x_{1}\left(-y_{3}\right) z_{2}<\left(-y_{1}\right) x_{2}\left(-z_{3}\right)$. Thus $x_{1} y_{3} z_{2}+x_{2} y_{1} z_{3}>0$. This, $k>0$ and $w>0$ imply the inequality here: $d_{1}+d_{2}+d_{3}+d_{4}=4 k+4 t=$ $4 w+4 x_{1} y_{3} z_{2}+4 x_{2} y_{1} z_{3}+4 k>0$.

Case 6. ++--- . We divide Case 6 into six subcases. In subcases 6.1-6.4 we consider $d_{3}+d_{4}$ and we deal with the value $h=-\left(k_{1}-1\right) x_{2} y_{3}-\left(k_{2}-1\right) x_{1} z_{3}+x_{1} y_{3}+$ $x_{2} z_{3}$. We always show that $h \geqslant 0$. This leads to the conclusion that $d_{3}+d_{4}>0$. Since we have $d_{3}+d_{4}=-2 p+2 k+2 t=-2 k_{3} y_{1}-2 k_{3} z_{2}-2\left(k_{3}-1\right) y_{1} z_{2}+2 x_{1} y_{3} z_{2}+$ $2 x_{2} y_{1} z_{3}+2 h+2 k>0$.

From (5) we conclude that $k_{1}+k_{2}-k_{1} k_{2}=k_{1} k_{2}\left(k_{1}^{-1}+k_{2}^{-1}-1\right)<0$. We shall apply this inequality in Subcases 6.3-6.6.

SUBCASE 6.1. When $\left(k_{1}-1\right)^{-1} x_{1} \leqslant x_{2} \leqslant\left(k_{2}-1\right) x_{1}$. We have $h=y_{3}\left[x_{1}-\right.$ $\left.\left(k_{1}-1\right) x_{2}\right]+z_{3}\left[x_{2}-\left(k_{2}-1\right) x_{1}\right] \geqslant 0$.

SUBCASE 6.2. When $\left(k_{1}-1\right) y_{3} \leqslant z_{3} \leqslant\left(k_{2}-1\right)^{-1} y_{3}$. Similarly to the preceding subcase $h=x_{1}\left[y_{3}-\left(k_{2}-1\right) z_{3}\right]+x_{2}\left[z_{3}-\left(k_{1}-1\right) y_{3}\right] \geqslant 0$.

SUBCASE 6.3. When $x_{2} \geqslant\left(k_{2}-1\right) x_{1}$ and $z_{3} \geqslant\left(k_{2}-1\right)^{-1} y_{3}$. There exists $\lambda \geqslant k_{2}-1$ such that $y_{3}=\lambda z_{3}$. We have $h=x_{1} y_{3}+x_{2} z_{3}-\left(k_{1}-1\right) \lambda x_{2} z_{3}-\left(k_{2}-1\right) x_{1} z_{3}=x_{1} y_{3}+$ $x_{2} z_{3}\left[1-\left(k_{1}-1\right) \lambda\right]-\left(k_{2}-1\right) x_{1} z_{3} \geqslant x_{1} y_{3}+\left(k_{2}-1\right) x_{1} z_{3}\left[1-\left(k_{1}-1\right) \lambda\right]-\left(k_{2}-1\right) x_{1} z_{3}=$ $x_{1} y_{3}-\left(k_{1}-1\right)\left(k_{2}-1\right) \lambda x_{1} z_{3}=x_{1} y_{3}-\left(k_{1}-1\right)\left(k_{2}-1\right) x_{1} y_{3}=\left(k_{1}+k_{2}-k_{1} k_{2}\right) x_{1} y_{3} \geqslant 0$.

SUBCASE 6.4. When $x_{2} \leqslant\left(k_{1}-1\right)^{-1} x_{1}$ and $z_{3} \leqslant\left(k_{1}-1\right) y_{3}$. There exists $\lambda \geqslant k_{1}-1$ such that $z_{3}=\lambda y_{3}$. We have $h=x_{1} y_{3}+x_{2} z_{3}-\left(k_{1}-1\right) x_{2} y_{3}-\left(k_{2}-1\right) x_{1} \lambda y_{3}=x_{1} y_{3}[1-$ $\left.\left(k_{2}-1\right) \lambda\right]+x_{2} z_{3}-\left(k_{1}-1\right) x_{2} y_{3} \geqslant\left(k_{1}-1\right) x_{2} y_{3}\left[1-\left(k_{2}-1\right) \lambda\right]+x_{2} z_{3}-\left(k_{1}-1\right) x_{2} y_{3}=$ $-x_{2} z_{3}\left(k_{1}-1\right)\left(k_{2}-1\right) \lambda+x_{2} z_{3}=\left(k_{1}+k_{2}-k_{1} k_{2}\right) x_{2} z_{3} \geqslant 0$.

SUBCASE 6.5. When $x_{2} \geqslant\left(k_{2}-1\right) x_{1}$ and $z_{3} \leqslant\left(k_{1}-1\right) y_{3}$. Consider the function $f(u, v)=k_{1} u-k_{2} v+u v$ for $u \in[0,1]$ and $v \in[-1,0]$. Looking at the partial derivatives, we deduce that if $u_{1} \geqslant u_{2}$ and $v_{1} \leqslant v_{2}$, then $f\left(u_{1}, v_{1}\right) \geqslant f\left(u_{2}, v_{2}\right)$. Thus $k_{1} x_{2}-k_{2} z_{3}+x_{2} z_{3} \geqslant k_{1}\left(k_{2}-1\right) x_{1}-k_{2}\left(k_{1}-1\right) y_{3}+\left(k_{1}-1\right)\left(k_{2}-1\right) x_{1} y_{3}$. Moreover, from the assumption of our subcase we get $-\left(k_{1}-1\right) x_{2} y_{3} \geqslant-\left(k_{1}-1\right)\left(k_{2}-1\right) x_{1} y_{3}$ and $-\left(k_{2}-1\right) x_{1} z_{3} \geqslant-\left(k_{1}-1\right)\left(k_{2}-1\right) x_{1} y_{3}$. In view of the above three inequalities 
we obtain $\left(-k_{2} x_{1}+k_{1} y_{3}+x_{1} y_{3}\right)+\left(k_{1} x_{2}-k_{2} z_{3}+x_{2} z_{3}\right)-\left(k_{1}-1\right) x_{2} y_{3}-\left(k_{2}-1\right) x_{1} z_{3}$ $\geqslant-k_{2} x_{1}+k_{1} y_{3}+x_{1} y_{3}+k_{1}\left(k_{2}-1\right) x_{1}-k_{2}\left(k_{1}-1\right) y_{3}+\left(k_{1}-1\right)\left(k_{2}-1\right) x_{1} y_{3}+\left(k_{1}-1\right)$ $\left(k_{2}-1\right) x_{1} y_{3}-2\left(k_{1}-1\right)\left(k_{2}-1\right) x_{1} y_{3}=\left(k_{1} k_{2}-k_{1}-k_{2}\right)\left[x_{1}\left(1-y_{3}\right)-y_{3}\right] \geqslant 0$. Hence $d_{4}=\left(-k_{2} x_{1}+k_{1} y_{3}+x_{1} y_{3}\right)+\left(k_{1} x_{2}-k_{2} z_{3}+x_{2} z_{3}\right)-\left(k_{1}-1\right) x_{2} y_{3}-\left(k_{2}-1\right) x_{1} z_{3}-$ $\left(k_{3}-1\right)\left[\left(y_{1}+1\right) z_{2}+y_{1}\right]-\left(1+x_{1} y_{3}\right) z_{2}+\left(x_{2}-1\right) y_{1}\left(z_{3}+1\right)-x_{1} z_{2}+y_{3} z_{2}+k$ is positive.

SUBCASE 6.6. When $x_{2} \leqslant\left(k_{1}-1\right)^{-1} x_{1}$ and $z_{3} \geqslant\left(k_{2}-1\right)^{-1} y_{3}$. We can write the assumptions of this subcase as $x_{1} \geqslant\left(k_{1}-1\right) x_{2}$ and $y_{3} \leqslant\left(k_{2}-1\right) z_{3}$. Then the considerations of the preceding case can be repeated for $d_{3}$ instead of $d_{4}$.

In the course of the proof of Theorem 2, we have shown that if $k_{1}^{-1}+k_{2}^{-1}+k_{3}^{-1}<1$, then the interior of the octahedron $Q$ with the vertices $x, y, z,-x,-y,-z$ contains at least one vertex of $P$. We formulate this as the first statement in the following Corollary 2. We change the notation in order to unify it with the notation of the forthcoming Conjecture 2. The second statement of Corollary 2 easily follows from the first one.

COROLlary 2. Let $Q$ be the octahedron with vertices $p_{1}=\left(p_{11}, p_{12}, p_{13}\right), p_{2}=$ $\left(p_{21}, p_{22}, p_{23}\right), \quad p_{3}=\left(p_{31}, p_{32}, p_{33}\right),-p_{1},-p_{2},-p_{3}$ such that $p_{i i} \geqslant 1$ for $i=1,2,3$ and $\left|p_{i j}\right| \leqslant 1$ for $i \neq j$. If $p_{11}^{-1}+p_{22}^{-1}+p_{33}^{-1}<1$, then the interior of $Q$ contains at least one point $\left(e_{1}, e_{2}, e_{3}\right)$, where $e_{1}, e_{2}, e_{3} \in\{-1,1\}$. Moreover, the weak inequality implies that just $Q$ contains at least one such a point.

More generally, consider the crosspolytope $Q=\operatorname{conv}\left\{p_{1}, p_{1}^{\prime}, \ldots, p_{n}, p_{n}^{\prime}\right\}$ in $E^{n}$, where $p_{i}=\left(p_{i 1}, \ldots, p_{i n}\right)$ and $p_{i}^{\prime}=\left(p_{i 1}^{\prime}, \ldots, p_{i n}^{\prime}\right)$ such that $p_{i i} \geqslant 1, p_{i i}^{\prime} \leqslant-1$ for $i=1 \ldots n$, and such that for every different $i, j \in\{1, \ldots n\}$ the inequality $\left|p_{i j}\right| \leqslant 1$ holds true.

The relationship between Theorem 2 and Corollary 2 can be expressed in the more general form: the conjecture that for every convex body $C$ and every parallelotope $P$ inscribed in $C$ we have $\sum_{i=1}^{n} k_{i}^{-1} \geqslant 1$ is equivalent to Conjecture 2 below.

Conjecture 2. If $\sum_{i=1}^{n}\left(p_{i i}-p_{i i}^{\prime}\right)^{-1}<1$, then the interior of the crosspolytope $Q$ contains at least one point of the form $\left(e_{1}, \ldots, e_{n}\right)$, where $e_{i} \in\{-1,1\}$ for $i=1, \ldots n$.

Let us show the equivalence by contradiction.

Assume that there exists a convex body $C$ and a parallelotope $P$ inscribed in $C$ such that $\sum_{i=1}^{n} k_{i}^{-1}<1$. We may assume that $\left(e_{1}, \ldots, e_{n}\right)$ are the vertices of $P$. (We just apply an affine transformation.) Support $C$ by $2 n$ hyperplanes parallel to the facets of $P$. Denote by $p_{i}$ and $p_{i}^{\prime}$ the points of support in $C \cap K_{i}$ such that $p_{i i} \geqslant 1$ and $p_{i i}^{\prime} \leqslant-1$. Since $P$ is inscribed in $C$ and since $Q \subset C$, no vertex of $P$ in the interior of $Q$. So Conjecture 2 is false. 
On the other hand, if Conjecture 2 is false, then there are points $p_{1}, p_{1}^{\prime}, \ldots, p_{n}, p_{n}^{\prime}$ with $p_{i i} \geqslant 1, p_{i i}^{\prime} \leqslant-1$ for $i=1, \ldots, n$ and with $\left|p_{i j}\right| \leqslant 1$ for $i \neq j$ such that $\sum_{i=1}^{n}\left(p_{i i}-p_{i i}^{\prime}\right)^{-1}<1$ and such that the interior of $Q$ does not contain all points of the form $\left(e_{1}, \ldots, e_{n}\right)$, where $e_{i} \in\{-1,1\}$. Let $C$ be the convex hull of the set of points consisting from all the $2^{n}$ points $\left(e_{1}, \ldots, e_{n}\right)$ and of points $p_{1}, p_{1}^{\prime}, \ldots, p_{n}, p_{n}^{\prime}$. Of course, $P$ is inscribed in $C$ but $\sum_{i=1}^{n} k_{i}^{-1}<1$.

From this equivalence and from the inequalities $k_{i} \leqslant w_{i}$ we conclude that Conjecture 1 is stronger than Conjecture 2.

It is easy to show that Conjecture 2 is equivalent to the following related conjecture: $\sum_{i=1}^{n}\left(p_{i i}-p_{i i}^{\prime}\right)^{-1} \leqslant 1$ implies that the crosspolytope $Q$ contains at least one point of the form $\left(e_{1}, \ldots, e_{n}\right)$, where $e_{i} \in\{-1,1\}$.

\section{REFERENCES}

[1] W. Boratyniski, 'Approximation of convex bodies by parallelotopes', in Proc. 7th Internat. Conference on Engin. Comput. graphics and Descriptive Geometry, Vol. 1-2 (Cracow, July 18-22, 1996), pp. 259-260.

[2] M. Lassak, 'Approximation of convex bodies by rectangles', Geom. Dedicata 47 (1993), 111-117.

[3] P.R. Scott, 'Lattices and convex sets in space', Quart. J. Math. Oxford (2) 36 (1985), 359-362.

[4] P.R. Scott, 'Properties of axial diameters', Bull. Austral. Math. Soc. 39 (1989), 329-333.

[5] J.M. Wills, 'Minkowski's inner and outer quermasses and convex bodies without interior lattice points', Quart. J. Math. Oxford (2) 41 (1990), 509-511.

Instytut Matematyki i Fizyli ATR

Bydgoszcz 85-796

Poland

e-mail: lassak@atr.bydgoszcz.pl 